\title{
Enhanced Glucose Requirement in Human Hepatoma-derived HuH-7 Cells by Forced Expression of the bcl-2 Gene
}

\author{
Kyoko Okamoto, Takashi Muraguchi, and Yoshihiro Shidoji* \\ Molecular \& Cellular Biology, Graduate School of Human Health Sciences, Siebold University of Nagasaki, \\ Academy Hills 1-1-1, Nagayo, Nagasaki 851-2195, Japan
}

Received 28 February, 2008; Accepted 11 March, 2008

\begin{abstract}
Summary To explore the metabolic effects of Bcl-2 in tumor cells, a stable clone of $\mathrm{HuH}-7 /$ bcl-2 and its control HuH-7/neo were established. Mitochondrial localization of ectopic Bcl-2 was demonstrated both by western blotting and immunofluorescence. HuH-7/bcl-2 cells consumed glucose at a higher rate, exhausted the available cellular ATP and died on day 9, while $\mathrm{HuH}-7 /$ neo cells were still alive for 10 days under the same condition where cells were cultured without replenishment of the medium. The expression of the hexokinase II gene was up-regulated in HuH-7/bcl-2 at its protein level. Taken together, we suggest that the forced expression of Bcl-2 in human hepatoma may cause the cells to become more glucose-dependent for survival.
\end{abstract}

Key Words: hepatoma, glycolysis, bcl-2, mitochondrial respiration, hexokinase II

\section{Introduction}

Otto Warburg originally proposed a hypothesis for the energy metabolism of tumor cells that the driving force of the enhanced glycolysis in hepatoma cells was the energy deficiency caused by an irreversible damage of the mitochondrial function [1]. There appears to be a diminished oxidative phosphorylation in most tumor cell types, including hepatoma, from the observations that numerous cancer cells display mitochondrial DNA deletions, reduced mitochondrial content, and impaired oxidative capacity [2]. Following Warburg, a number of these explanations have been advocated for experimental tumor cells; however, a common molecular mechanism of a deficit in oxidative metabolism has proven still elusive [3].

It is well established that activated oncogenes play pivotal roles in the tumorigenesis of most tumor types. In particular, the up-regulation of the oncogenes $c-m y c$, ras, or $b c l-2$, is a

\footnotetext{
*To whom correspondence should be addressed.

Tel/Fax: +81-95-813-5207

E-mail: shidoji@sun.ac.jp
}

prevalent feature in various human cancers [4]. Among these 3 genes, the metabolic roles of $c-m y c$ have so far been most successfully investigated, and the c-Myc has been demonstrated to transactivate the lactate dehydrogenase A $(\mathrm{LDH}$ A) gene [5], whose product participates in anaerobic glycolysis, suggesting that the high glycolytic rate in tumor cells could be explained by the activation of the $c-m y c$ gene. However, the Warburg's theory of irreversible damage to mitochondrial function is still difficult to account for by the $c$-myc gene alone. The other 2 oncogenes have not been shown to induce mitochondrial malfunction in respiration.

The mitochondrion has been recognized mainly as an organelle with the functions of a cellular power plant, which produces energy for cell to survive [6]. However, a recent review has extensively described an additional role that mitochondria play role in cell fate [7]. Apoptotic cell death is a highly controlled process under the control of certain gene products. Bcl-2 is well known to prevent apoptosis by blocking the cyotosolic release of cytochrome $c$ from mitochondria [7].

We have focused our attention to the $b c l-2$ oncogene, which is up-regulated in more than $50 \%$ of human tumor types and the product of which is localized mainly to the 
mitochondrial outer membrane [7]. In the present study we established a stable cell clone from human hepatomaderived HuH-7 cells, HuH-7/bcl-2, which exhibited constitutive expression of the $b c l-2$ gene, as well a negative control clone, HuH-7/neo. The mitochondrial localization of Bcl-2 forcibly expressed was confirmed both by subcellular fractionation followed by western blotting, and immunofluorescence with confocal laser scanning microscopy. We found that $\mathrm{HuH}-7 / \mathrm{bcl}-2$ cells consumed glucose more rapidly than $\mathrm{HuH}-7 /$ neo cells in a high glucose medium, suggesting that the forced expression of the bcl-2 gene in human hepatoma may make the cells more glucose-dependent.

\section{Materials and Methods}

\section{Cells}

Human hepatoma-derived HuH-7 cells were obtained from RIKEN BioResource Center, Tsukuba, Japan and were grown in the presence of $5 \% \mathrm{CO}_{2}$ with Dulbecco's modified Eagle's (DME) medium containing high glucose $(25 \mathrm{mM}$, Sigma-Aldrich, St. Louis, MI) with 5\% fetal bovine serum (FBS, Hyclone, Logan, UT). DME medium containing low glucose (5.5 mM, Sigma-Aldrich) was also used.

\section{Plasmids and stable transfection}

An Eco RI fragment of pCMV/bcl-2 (a generous gift from Dr. Y. Akao, Gifu International Institute of Bioscience), was subcloned into the Eco RI site of pcDNA3.1(+) (Invitrogen, CA). HuH-7 cells were transfected with the $B g l$ II-linearized pcDNA3.1/bcl-2 or the empty vector, pcDNA3.1(+). Transfected clones were selected by resistance to G418 (150 $\mu \mathrm{g} /$ $\mathrm{ml}$, Invitrogen) in 6-well plates with a cylinder cloning method.

\section{Cell proliferation assay}

One thousand cells were inoculated per well in a 96-well culture plate. Cells were detected using the Cell Proliferation Reagent WST-1 for cell proliferation according to the protocols of the manufacturer (Roche Diagnostics, Tokyo). Ten $\mu 1 /$ well of WST-1 were added, and incubated for $30 \mathrm{~min}$. Absorbance was measured in a HTS7000 plate reader (Hewlett Packard, Palo Alto, CA) at $450 \mathrm{~nm}$ with a subtraction of the cell density background at $690 \mathrm{~nm}$. The experiment was performed in triplicate.

\section{Cellular ATP contents}

At the indicated time of culture, cellular ATP content was evaluated using the CellTiter-Glo ${ }^{\circledR}$ Luminescent Cell Viability Assay (Promega, Madison, WI) according to the manufacturer's instructions. The intensity of luminescence was measured in a Centro XS ${ }^{3}$ LB 960 (Berthold Technology GmbH \& Co KG, Germany). The experiment was performed in triplicate.

\section{Real-time RT-PCR}

Real time PCR was performed to quantify the cellular levels of hexokinase type 2 (HK II) mRNA, in comparison with those of glucokinase (GK) mRNA. The primers for HK II were: HK II fw: 5'-ATC CCT GAG GAC ATC ATG CGA3' and HK II rev: 5'-CTT ATC CAT GAA GTT AGC CAG GCA-3'. For GK: GK fw 5'-CTC CCA AAG CAT CTA CCT CT-3' and GK rev: 5'-TCC TTC TGC ATC CGT CTC AT-3' were used.

Amplification in the LightCycler capillaries (Roche, Tokyo) was for 40 cycles with initial incubation of $10 \mathrm{~min}$ at $95^{\circ} \mathrm{C}$ for activation of Taq DNA polymerase in LightCycler FastStart DNA Master ${ }^{\text {PLus }}$ SYBR Green I (Roche Diagnostics, Indianapolis, IN). Cycling parameters were $15 \mathrm{~s}$ at $95^{\circ} \mathrm{C}, 10 \mathrm{~s}$ at $60^{\circ} \mathrm{C}$, and $10 \mathrm{~s}$ at $72^{\circ} \mathrm{C}$. The second derivative maximum method was used for quantification with the LightCycler software (Roche Diagnostics). For quantification of the results, normalization was performed by dividing each value calculated for GK or HK II mRNAs in $\mathrm{HuH}-7 /$ neo cells.

\section{Subcellular fractionation}

Subcellular fractionation was conducted prior to western blotting. Cells were scraped in Dulbecco's phosphate buffered saline, $\mathrm{Ca}^{2+}$ free [PBS (-)] and centrifuged at $1,000 \times \mathrm{g}$ for $5 \mathrm{~min}$. The pelleted cells were washed with $0.13 \mathrm{M} \mathrm{NaCl}, 5 \mathrm{mM} \mathrm{KCl}$, and $1 \mathrm{mM} \mathrm{MgCl}$. The washed cells were suspended in $0.01 \mathrm{M}$ Tris- $\mathrm{HCl}$ buffer ( $\mathrm{pH}$ 6.7) containing $0.01 \mathrm{M} \mathrm{KCl}$ and $0.1 \mathrm{mM}$ EDTA. After homogenization in $0.2 \mathrm{M}$ sucrose, the cell debris were removed by centrifugation. The supernatants were centrifuged at $8,300 \times \mathrm{g}$ for $19 \mathrm{~min}$; and the pellet was resuspended in $0.25 \mathrm{M}$ sucrose in $0.01 \mathrm{M}$ Tris- $\mathrm{HCl}, \mathrm{pH} 6.7$, containing $0.1 \mathrm{mM}$ EDTA, which was referred to as "the mitochondrial fraction". The remaining supernatants were centrifuged at $16,700 \times \mathrm{g}$ for $19 \mathrm{~min}$; and the resultant supernatants were saved as "the post-mitochondrial fraction" or, tentatively, "the cytosolic fraction".

\section{Western blotting}

After being washed with PBS (-), the cells were lysed on ice in RIPA buffer containing complete ${ }^{\mathrm{TM}}$ Mini (protease inhibitor cocktail, Roche Diagnostics). The mitochondrial fractions ( $5 \mu \mathrm{g}$ total protein) or the post-mitochondrial fractions $(10 \mu \mathrm{g})$ were heated at $95^{\circ} \mathrm{C}$ for $5 \mathrm{~min}$, and applied to a $15 \%$ polyacrylamide gel containing $1 \%$ SDS. The proteins were semi-dry blotted onto PVDF membrane (Bio-Rad) and were incubated with polyclonal anti-bcl-2 antibody $(\triangle \mathrm{C} 21$, Santa Cruz, 1:400) or anti-hexokinase II (HK II, 1:1000, Cell Signaling, Danvers, MA) at $4^{\circ} \mathrm{C}$ overnight. Anti-rabbit$\mathrm{IgG}$ antibody labeled with horseradish peroxidase (Santa Cruz, 1:800) was detected with ECL plus reagent (GE Healthcare UK, England). The chemiluminescence was 
A

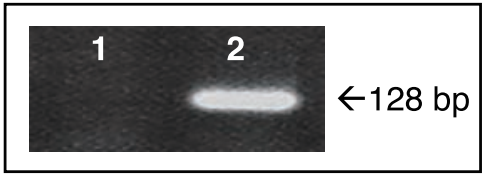

B

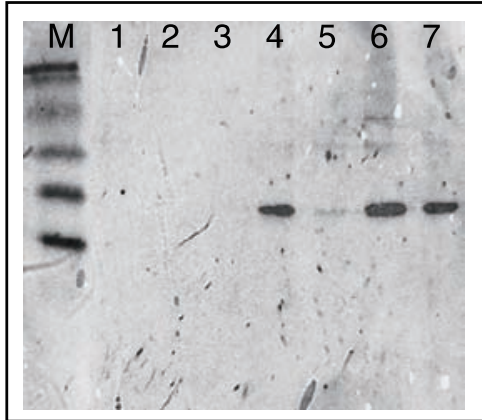

C

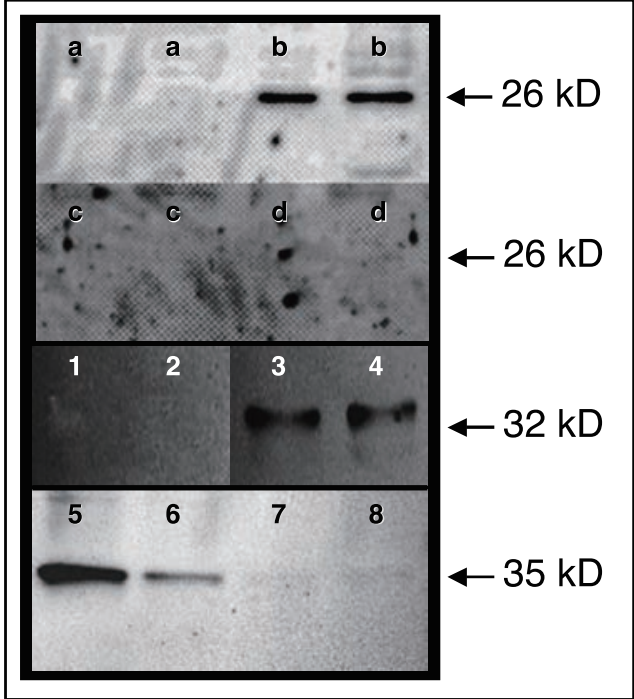

$\mathbf{D}$
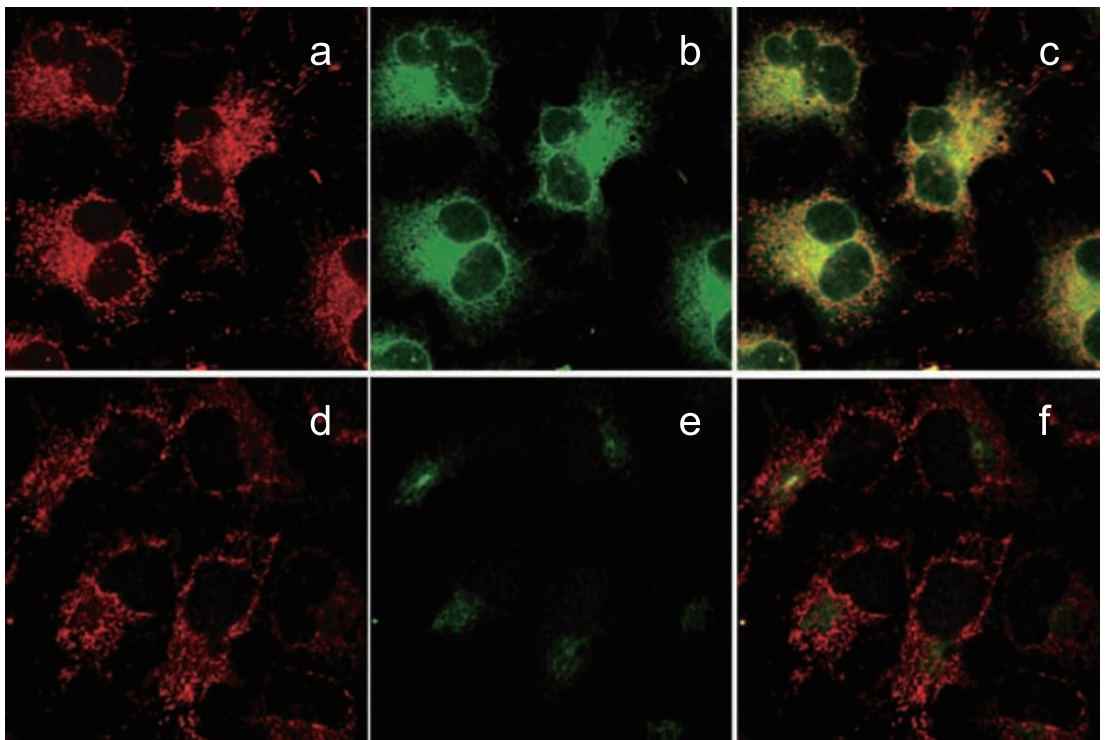

Fig. 1. The expression of the $b c l-2$ gene at its transcriptional and translational levels and mitochondrial localization of Bcl-2 protein in HuH-7/bcl-2 cells. A: RT-PCR of the bcl-2 gene from total RNA in HuH-7/neo (lane 1) or HuH-7/bcl-2 cells (lane 2). B: Immunoblot of whole cell lysates with anti-bcl-2 antibody in each $3 \mathrm{HuH}-7 /$ neo cell clones (lanes 1, 2, 3), and each $4 \mathrm{HuH}-7 /$ bcl-2 cell clones (lanes 4, 5, 6, 7). Lane $M$ shows molecular weight standard proteins containing 20, 28, 35, 45, 56, and 75 kD. $\mathrm{C}$ : Immunoblots with anti-bcl-2 antibody of the mitochondrial fractions (lanes $a, b$ ) and the post-mitochondrial fractions (lanes $c, d$ ) from HuH-7/neo (lanes $a, c$, in duplicate) and HuH-7/bcl-2 cells (lanes $b, d$ in duplicate). Immunoblots with anti-porin antibody of the post-mitochondrial fractions (lanes 1,2) and the mitochondrial fractions (lanes 3, 4) from HuH-7/neo (lanes 1, 3) and $\mathrm{HuH}-7 / \mathrm{bcl}-2$ (lanes 2, 4), and with anti-LDH-A antibody of the post-mitochondrial fractions (lanes 5, 6) and the mitochondrial fractions (lanes 7, 8) from HuH-7/neo (lanes 5, 7) and HuH-7/bcl-2 (lanes 6, 8). D: Immunofluorescence of Bcl-2 was observed by a laser-scanning microscopy. Red fluorescence indicates mitochondria with MitoTracker Red and green fluorescence displays the distribution of $\mathrm{Bcl}-2$ in $\mathrm{HuH}-7 / \mathrm{bcl}-2(a, b, c)$ or HuH-7/neo cells $(d, e, f)$. 
recorded with a LAS 1000plus Intelligent Dark Box II (Fujifilm, Tokyo). Anti-porin (Calbiochem, Merck, Darmstadt, Germany, 1:1000) and anti-LDH (Chemicon, CA, 1:2500) were used to detect marker proteins for mitochondria and cytosol fractions, respectively.

\section{Immunofluorescence}

Cells were incubated for $30 \mathrm{~min}$ with $100 \mathrm{nM}$ MitoTrackerRed CMXRos (Molecular Probe, Eugene, OR) in FBS-free DME medium. The cells were then fixed with $4 \%$ paraformaldehyde containing $2 \%$ sucrose in PBS (-) for $30 \mathrm{~min}$. After incubated with anti-bcl-2 antibody $(1: 400)$ at $4{ }^{\circ} \mathrm{C}$, overnight, the cells were incubated for $2.5 \mathrm{~h}$ at room temperature with anti-rabbit-IgG-Alexa-488 (Molecular Probe, 1:1000). The cells mounted in ParmaFlour were observed under a confocal laser-scanning fluorescence microscope, LSM 510 equipped with Axiovert 200M (Carl Zeiss, Germany).

\section{Results}

\section{Mitochondrial localization of Bcl-2 in $\mathrm{HuH}-7 / \mathrm{bcl}-2$ cells}

To establish the transfected clones, the expression of the bcl-2 gene was detected by a RT-PCR method. Fig. 1A clearly shows the ectopic expression of the $b c l-2$ gene in $\mathrm{HuH}-7 / \mathrm{bcl}-2$ cells, while no transcript was detected in $\mathrm{HuH}-$ 7/neo cells. Furthermore, Bcl-2 was detected on western blot with the RIPA lysates of 4 individual clones of $\mathrm{HuH}-7 / \mathrm{bcl}-2$, but not in any of the 3 clones of HuH-7/neo (Fig. 1B). Thereafter we used clone 11 (lane 6 in Fig. 1B) of HuH-7/bcl-2 for further experimentation in the present study. Bcl-2 was mainly found in mitochondria and barely detected in the post-mitochondrial fraction (Fig. 1C). The mitochondrial localization of $\mathrm{Bcl}-2$ was also demonstrated by 2-color immunofluorescence with confocal laser scanning microscopy. Fig. 1D illustrates the co-localization of Bcl-2-specific green fluorescence with red fluorescence of MitoTracker in $\mathrm{HuH}-7 / \mathrm{bcl}-2$ cells, but scarce green fluorescence specific for Bcl-2 was found in $\mathrm{HuH}-7 /$ neo cells.

\section{Growth kinetics of HuH-7/bcl-2 and HuH-7/neo cells}

The effects of constitutive expression of the $b c l-2$ gene on growth were examined with the WST-1 assay. There was no significant difference in the standard curves of WST-1 colorization vs. cell number, as measured by the Trypan blue method, between either the HuH-7/neo and $\mathrm{HuH}-7 / \mathrm{bcl}-2$ cells (data not shown). As shown in Fig. 2A, both HuH-7/ neo and HuH-7/bcl-2 cells started to grow similarly in a logarithmic mode, and there was no difference in the growth rate of these 2 cell lines. The both cell populations continued to expand and reached a maximum density on day 7 . The HuH-7/bcl-2 cells suddenly died on day 8 , whereas most of the HuH-7/neo cells were still alive on day 10 (Fig. 2A).

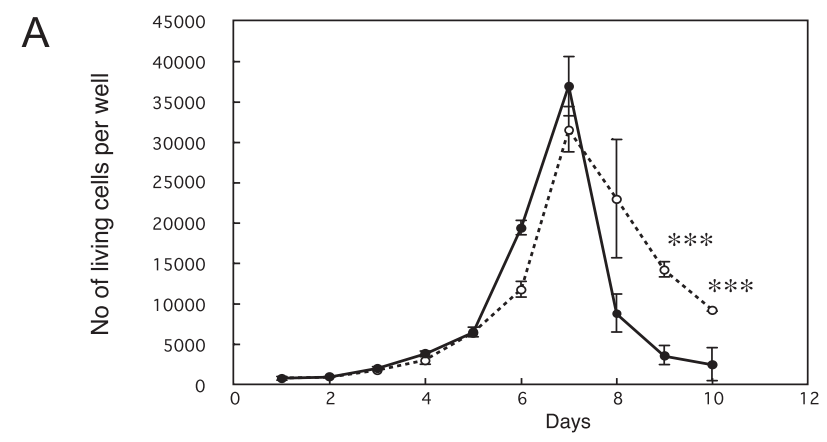

B

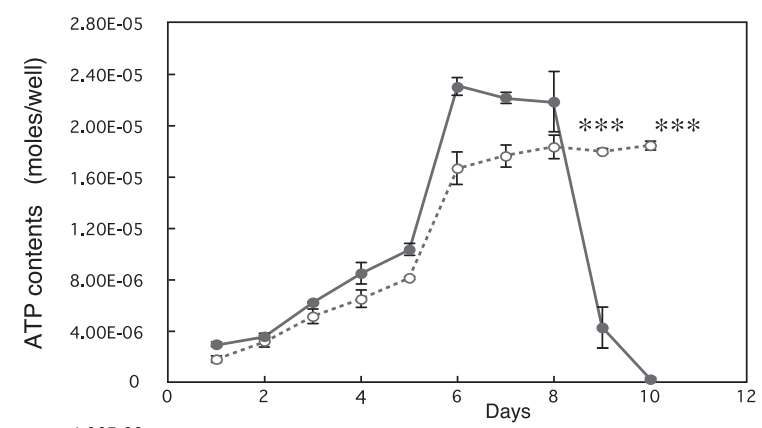

C

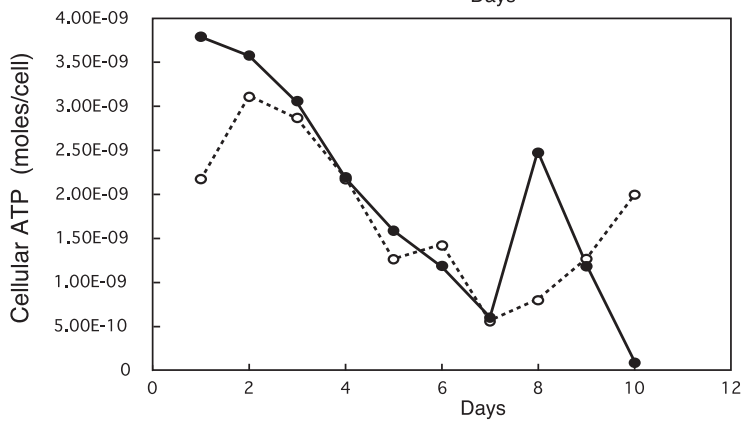

Fig. 2. Growth curves of HuH-7/bcl-2 and HuH-7/neo cells revealed by WST-1 assay and changes in ATP contents per well. HuH-7/neo (open circle) and HuH-7/bcl-2 cells (closed circle) were cultured with $25 \mathrm{mM}$ glucose. A: Numbers of living cells measured with WST-1 were plotted per well against days in culture. B: ATP concentrations (moles per well) the CellTiter Glo assay. Each points represent mean $\pm \mathrm{SD}(n=3)$. *: $p<0.01$, $* * *: p<0.005$. C: Cellular ATP concentrations (moles per cell) were plotted from a calculation of the each value in panel $\mathrm{B}$ divided by the corresponding cell number in panel A.

Next we determined the number of viable cells in a 96well plate based on quantitation of the cellular ATP present, which signals the presence of metabolically active cells. As shown in Fig. 2B, ATP-contents per well reached a plateau on day 6 with the HuH-7/bcl-2 cells, then they suddenly declined and almost disappeared on day 9, while the ATPcontents of HuH-7/neo cells per well maintained their plateau level until the end of the experiment. When we plotted time-dependent changes in the ATP levels at a cellular basis (Fig. 2C), both cells gradually and continu- 


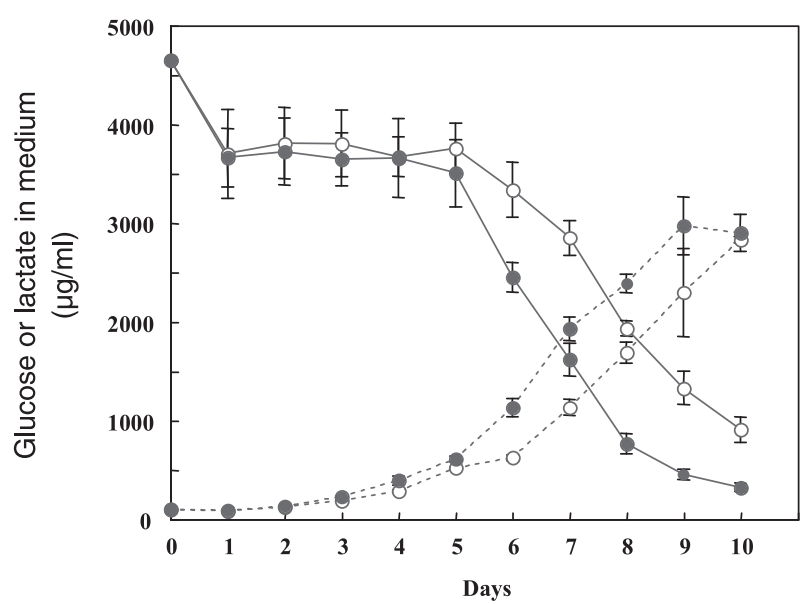

Fig. 3. Glucose consumption and lactate production in HuH-7/ neo and $\mathrm{HuH}-7 / \mathrm{bcl}-2$ cells. HuH-7/neo (open circle) and HuH-7/bcl-2 (closed circle) was cultured with $25 \mathrm{mM}$ glucose. Concentrations $(\mu \mathrm{g} / \mathrm{ml})$ of glucose (continuous line) and lactate (broken line) in medium were plotted against days in culture. Each points represent mean \pm SD $(n=3)$.

ously decreased their ATP contents until day 7. From day 8 to day $10, \mathrm{HuH}-7 /$ neo cells restored the cellular ATP contents almost up to the initial level, whereas $\mathrm{HuH}-7 / \mathrm{bcl}-2$ cells exhausted all cellular ATP via its apparent transient increase.

\section{Enhanced glucose consumption by the forced expression of the bcl-2 gene}

Both cell lines took up large amounts of the glucose in the medium on day 1 . And the concentrations of glucose were the same with both cell lines from day 2 to day 5 (Fig. 3 ). Then, on day 6 , HuH-7/bcl-2 cells restarted to uptake glucose and continued to consume it until day 10, whereas HuH-7/ neo cells restarted to uptake glucose on day 7 , one day later than the $\mathrm{HuH}-7 / \mathrm{bcl}-2$ cells. Concomitantly, lactate was secreted and accumulated 1 day earlier in the HuH-7/bcl-2 cells than the HuH-7/neo cells (Fig. 3). The day when the disappearance curve of glucose and production curve of lactate in the medium crossed over was day 7 for $\mathrm{HuH}-7 / \mathrm{bcl}-$ 2 , and day 8 for $\mathrm{HuH}-7 /$ neo cells, respectively.

The glucose consumption and lactate production rates were calculated as shown in Table 1 . HuH-7/bcl-2 cells utilized $65 \%$ of the consumed glucose only for the glycolysis pathway, whereas a nearly equal amount (2732.1 or $2800.2 \mu \mathrm{g} / \mathrm{ml}$ ) of lactate was produced in both cell lines. This indicates the over-expression of the $b c l-2$ gene in hepatoma cells induced extra energy metabolism of glucose in response to mitochondrial respiration in addition to keeping glycolysis to lactate at a high constant rate.

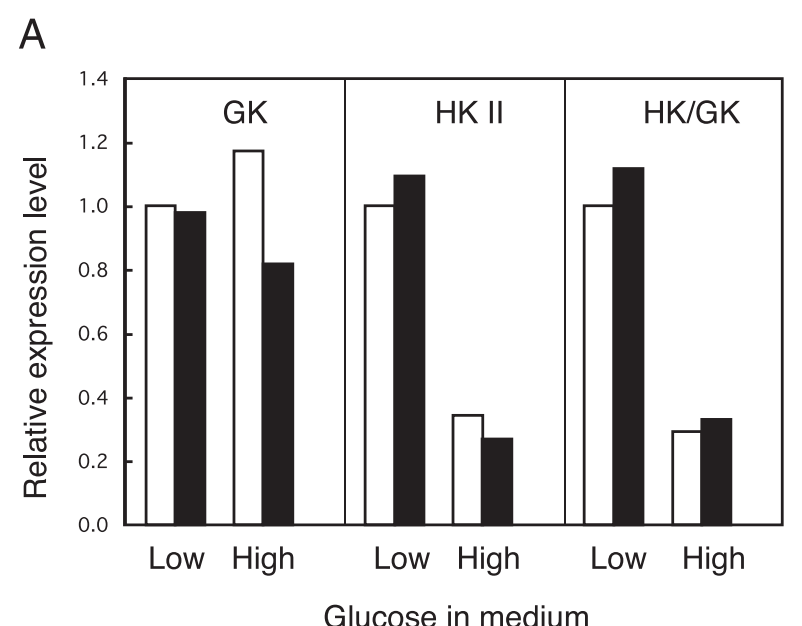

\section{B}

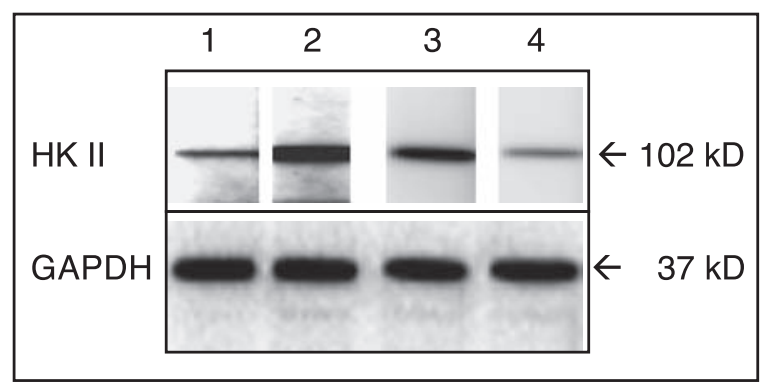

Fig. 4. Up-regulation of HK II expression by Bcl-2 and its down-regulation by high glucose. A: Real time RT-PCR analysis of HK II and GK mRNA expression was performed using total RNA isolated from $\mathrm{HuH}-7 /$ neo (open column) and HuH-7/bcl-2 cells (closed column). Data are normalized for the expression levels or their ratio of HuH-7/neo in low glucose. B: HK II protein was analyzed by immunoblot with loading control of GAP$\mathrm{DH}$ in whole cell lysates of HuH-7/neo (lanes 1,3) and HuH-7/bcl-2 cells (lanes 2, 4) cultured with low glucose (lanes 1,2) or high glucose (lanes 3, 4).

Enhanced expression of the hexokinase II (HK II) gene by the forced expression of the bcl-2 gene

Hexokinase is the initial enzyme in the glycolysis pathway that catalyzes the irreversible reaction of glucose to glucose-6-phosphate. In order to clarify a mechanism of the enhanced glucose consumption in $\mathrm{HuH}-7 / \mathrm{bcl}-2$ cells, the expression levels of the $H K I I$ and $G K$ genes were investigated. As shown in Fig. 4A, the cellular levels of $G K$ mRNA were not so altered by Bcl-2 in either low or high glucose medium. The cellular levels of $H K I I$ mRNA were also not so varied by Bcl-2, but were down-regulated by one-third in high glucose medium. Most interestingly, the cellular levels of HK II protein were highly up-regulated by Bcl-2 in low glucose medium, whereas high glucose media down- 
Table 1. Glucose consumption and lactate production in HuH-7/neo and HuH-7/bcl-2 cells during 10-day culture.

\begin{tabular}{lrr}
\hline & HuH-7/neo & HuH-7/bcl-2 \\
\hline Low Glucose $(5.5 \mathrm{mM})$ & & \\
Glucose consumed in total $(\mu \mathrm{g} / \mathrm{ml}):$ A & 1107.1 & 1106.0 \\
Lactate produced in total $(\mu \mathrm{g} / \mathrm{ml}): \mathrm{B}$ & 905.6 & 882.0 \\
B/A $\times 100(\%)$ & 81.8 & 79.7 \\
High Glucose $(25 \mathrm{mM})$ & & \\
Glucose consumed in total $(\mu \mathrm{g} / \mathrm{ml}):$ A & 3741.7 & 4326.5 \\
Lactate produced in total $(\mu \mathrm{g} / \mathrm{ml}): \mathrm{B}$ & 2732.1 & 2800.2 \\
B/A $\times 100(\%)$ & 73.0 & 64.7 \\
\hline
\end{tabular}

regulated HK II protein levels both in $\mathrm{HuH}-7 /$ neo and HuH-7/bcl-2 cells (Fig. 4B).

\section{Discussion}

In the present study, it was found the forced expression of the $b c l-2$ gene caused an enhanced glucose requirement of human hepatoma cells, suggesting that this oncogene apparently brought about by the typical characteristics of tumor cells.

In addition to preventing apoptotic cell death by inhibiting the secretion of proapoptotic factors from mitochondria, $\mathrm{Bcl}-2$ has recently been reported to be involved also in the energetics of mitochondria [ 6 ], an organelle where $\mathrm{Bcl}-2$ mainly resides $[8,9]$. To explore a possible metabolic effect of Bcl-2 on glucose-dependency of tumor cells, we established several stable clones of Bcl-2-expressing $\mathrm{HuH}-7$ by transfection with the $b c l-2$ gene. HuH-7/bcl-2 cells expressed Bcl-2 even after the 58th passage, and most of the protein was localized to mitochondria. Therefore, we utilized these 2 cell lines, HuH-7/neo and HuH-7/bcl-2, to investigate the cellular effects of $\mathrm{Bcl}-2$ on glucose metabolism and cell growth.

The most interesting finding in the present study is that in comparison with $\mathrm{HuH}-7 /$ neo cells, glucose utilization was enhanced in HuH-7/bcl-2 cells. Further HuH-7/bcl-2 cells were selectively killed due to depletion of cellular ATP after prolonged culture time. This means that when glucose is sufficiently available, a greater range of malignant cells, such as HuH-7/bcl-2 cells are able to utilize increased amounts of glucose for mitochondrial respiration than the differentiated tumor-type HuH-7/neo cells. However, the glycolysis rate in $\mathrm{HuH}-7 / \mathrm{bcl}-2$ cells might be equal to that of HuH-7/neo cells, because the production rate of lactate was similar in both cell lines (Table 1). This explanation of the finding would seem to be inconsistent with two long standing ideas in the oncology field, i.e. a "Warburg theory" and the "Crabtree effect" in tumor cells [1]. As is well known, Warburg proposed putative irreversible damage of mitochondrial function in most tumors [1]. In contrast, the present study strongly suggests that the enhanced expression of Bcl-2 might unexpectedly cause up-regulation of mitochondrial utilization of glucose metabolites, or respiration. Crabtree originally found that a high concentration of glucose indeed did inhibit mitochondrial respiration [1]. This "Crabtree effect" is a metabolic effect typical of tumor cells. Therefore, the above-mentioned scenario of constant glycolysis and up-regulated respiration by high concentration of glucose may require revision to account for why HuH-7 hepatoma cells apparently lack the Crabtree effect.

To our knowledge, only a couple of papers have so far reported an intracellular link between $\mathrm{Bcl}-2$ (or Bcl-xl) and glucose metabolism $[10,11]$. Tome et al. [10] found that mouse thymic lymphoma cells over-expressing Bcl-2 exhibited increased activity in hexokinase and lactate production, suggesting a potential increase in glycolysis. In the present study, we also found the up-regulation of HK II protein in HuH-7/bcl-2 cells only in low glucose condition, but failed to find any apparent increase in lactate production in HuH-7/bcl-2 cells. This may be due to a potential difference in intrinsic glucose metabolism between lymphoma and hepatoma cells. It has been well known that HK II protein is frequently up-regulated in most tumor cells and is bound to the outer mitochondrial membrane, whereas glucokinase is not up-regulated in hepatoma and is the cytosolic soluble protein [12]. However, the up-regulation of HK II by bcl-2 over-expression was limited to the low glucose condition. Therefore, we speculate that the overexpression of HK II protein may not be a major mechanism for the enhanced glucose requirement induced by bcl-2 overexpression.

In contrast, Panickar et al. [11] reported that overexpression of Bcl-xl protected neurons from prolonged hypoglycemic stress. The authors explained that the preventive effect of the over-expressed Bcl-xl was not on glucose metabolism, but rather on mitochondrial apoptosis, which was induced by hypoglycemic stress. However, an alternative explanation for the prolonged survival of Bcl-xl-over- 
expressing cells is that Bcl-xl promoted the mitochondrial respiratory function. This is because Bcl-xl and Bcl-2 are known to be involved in mitochondrial electron transport system by their interaction with voltage-dependent anion channels $[4,7]$. In fact, in the present study the enhanced glucose consumption by $\mathrm{HuH}-7 / \mathrm{bcl}-2$ cells was not due to an increased metabolic flow to lactate in glycolysis, but was due to an incremental change in metabolic flow from glucose to other pathways (Table 1).

Very recently, an interesting and important paper appeared on the engagement of Bcl-2 with mitochondrial respiration. Chen et al. [13] reported a higher level of mitochondrial respiration in tumor cells over-expressing $\mathrm{Bcl}-2$. In this case, the impact of Bcl-2 on mitochondrial respiration is apparently opposite to the Warburg theory, but is supporting of the present findings on the enhanced glucose consumption for the extra-glycolysis in Bcl-2expressing HuH-7 cells.

Most oncology scientists would agree that tumor cells are apparently devoid of the Pasteur effect, which says that an increase in oxygen pressure brings on a down-regulation of glycolysis [14]. In our experimental conditions, where cells were always exposed to normoxia or hyperoxia, no downregulation of glycolysis was observed in either cell line, indicating that HuH-7 cells, whether expressing Bcl-2 or not, indeed lacked the Pasteur effect.

Finally, it is worth noting that the cellular ATP content in cells grown with the higher glucose was consistently higher than in cells incubated with the lower glucose in both cell lines. The cellular ATP content $\left(0.5-7.5 \times 10^{-14}\right.$ moles/cell) measured in this study was in the range of cellular ATP concentrations of various cultured cells, including $\mathrm{HuH}-7$, found in the literature $[15,16]$. A recent review pointed out that the average contribution of glycolysis in cellular ATP production in tumor cells was not markedly different from that of normal cells [17].

In conclusion, the present study found that the forced expression of the $b c l-2$ gene in human hepatoma-derived HuH-7 cells enhanced glucose consumption when the cells were grown with high glucose medium. A possible molecular mechanism underlying the enhanced glucose requirement of the Bcl-2 expressing HuH-7 cells is favored in terms of the recently reported Bcl-2-enhanced mitochondrial respiration, because the expression of the $b c l-2$ gene in HuH-7 cells had apparently no effect on the rate of glycolysis.

\section{Acknowledgments}

The authors thank Dr. Luigi M. De Luca (Johns Hopkins University) for his kind advice during preparation of this manuscript.

\section{Abbreviations}

LDH-A, lactate dehydrogenase A; HK II, hexokinase type 2; FBS, fetal bovine serum; GK, glucokinase.

\section{References}

[1] Chance, B.: Was Warburg right? Or was it that simple? Cancer Biol. Ther., 4, 125-126, 2005.

[2] Moreno-Sanchez, R., Rodriguez-Enriquez, S., MarinHernandez, A., and Saavedra, E.: Energy metabolism in tumor cells. FEBS J., 274, 1393-1418, 2007.

[3] Shaw, R.J.: Glucose metabolism and cancer. Curr. Opinion Cell Biol., 18, 598-608, 2006.

[4] Letai, A.: Pharmacological manipulation of Bcl-2 family members to control cell death. J. Clin. Invest., 115, 26482655, 2005.

[5] Shim, H., Chun, Y., Lewis, B., and Dang, C.: A unique glucose-dependent apoptotic pathway induced by c-Myc. Proc. Natl. Acad. Sci. USA, 95, 1511-1516, 1998.

[6] Murphy, E., Imahashi, K., and Steenbergen, C.: Bcl-2 regulation of mitochondrial energetics. Trends Cardiovasc. Med., 15, 283-290, 2005.

[7] Kroemer, G., Galluzzi, L., and Brenner, C.: Mitochondrial membrane permeabilization in cell death. Physiol. Rev., 87, 99-163, 2007.

[8] Green, D.R. and Kroemer, G.: Pharmacological manipulation of cell death: clinical applications in sight?. J. Clin. Invest., 115, 2610-2617, 2005.

[9] Gustafsson, A.B. and Gottlieb, R.A.: Bcl-2 family members and apoptosis, taken to heart. Am. J. Physiol. Cell Physiol., 292, C45-C51, 2007.

[10] Tome, M.E., Lutzb, N.W., and Briehl, M.M.: Overexpression of catalase or Bcl-2 alters glucose and energy metabolism concomitant with dexamethasone resistance. Biochim. Biophys. Acta, 1693, 57-72, 2004.

[11] Panickar, K., Nonner, D., and Barrett, J.: Overexpression of Bcl-xl protects septal neurons from prolonged hypoglycemia and from acute ischemia-like stress. Neuroscience, 135, 73$80,2005$.

[12] Lee, M.G. and Pedersen, P.L.: Glucose metabolism in cancer. Importance of transcription factor-DNA interactions within a short segment of the proximal region of the type II hexokinase promoter. J. Biol. Chem., 278, 41047-41058, 2003.

[13] Chen, Z.X. and Pervaiz, S.: Bcl-2 induces pro-oxidant state by engaging mitochondrial respiration in tumor cells. Cell Death Different., 14, 1617-1627, 2007.

[14] Garber, K.: Energy boost: The Warburg effect returns in a new theory of cancer. J. Natl. Cancer Inst., 96, 1805-1806, 2004.

[15] Berson, A., Cazanave, S., Descatoir, V., Tinel, M., Grodet, A., Wolf, C., Feldmann, G., and Pessayre, D.: The antiinflammatory drug, nimesulide (4-nitro-2-phenoxymethanesulfoanilide), uncouples mitochondria and induces mitochondrial permeability transition in human hepatoma cells: 
protection by albumin. J. Pharmacol. Exp. Therapeutic., 318, 444-454, 2006.

[16] Rodriguez-Enriquez, S., Vital-Gonzalez, P.A., FloresRodriguez, F.L., Marin-Hermandez, A., Ruiz-Azuara, L., and Moreno-Sanchez, R.: Control of cellular proliferation by modulation of oxidative phosphorylation in human and rodent fast-growing tumor cells. Toxicol. Appl. Pharmacol., 215, 208-217, 2006.

[17] $\mathrm{Zu}$, X.L. and Guppy, M.: Cancer metabolism: facts, fantasy, and fiction. Biochem. Biophys. Res. Commun., 313, 459-465, 2004. 